PROCEEDINGS OF THE

AMERICAN MATHEMATICAL SOCIETY

Volume 49, Number 2, June 1975

\title{
LIFTING RECURSION PROPERTIES THROUGH GROUP HOMOMORPHISMS
}

\section{S. H. AL-KUTAIBI AND F. RHODES}

ABSTRACT. The problem of lifting recursion properties is studied for the case of homomorphisms between transformation groups with different acting groups. It is shown that if the spaces are the same and the group homomorphism is compact-covering then almost periodicity and recurrence lift. This result is applied to certain group actions on covering spaces and on fibre bundles with totally disconnected bundle groups induced by group actions on the base spaces. In particular, it is shown that under suitable conditions if a point in the base space of a fibre bundle with finite bundle group is $\{$ almost periodic $\}$ \{recurrent $\}$ under the action of a group $G$ then every point in the fibre over the given point is \{almost periodic\} \{recurrent\} under the induced action of a covering group $\widetilde{G}$.

1. Introduction. The problem of lifting dynamical properties through a transformation group homomorphism $(Y, G) \rightarrow(X, G)$ has received considerable attention. In this note we consider epimorphisms $(Y, H) \rightarrow(X, G)$ for transformation groups with different acting groups. There is an induced action of $H$ on $X$, and the epimorphism factors through $(X, H)$. Previous results can be used for lifting dynamical properties from $(X, H)$ to $(Y, H)$. Since the actions of $G$ and $H$ on $X$ when made effective are the same, dynamical properties which concern only the whole group such as distal and uniform equicontinuity clearly lift from $(X, G)$ to $(X, H)$. Thus we restrict our attention to recursion properties. In the next section we prove that if the map $H \rightarrow G$ is compact-covering then almost periodicity and recurrence lift from $(X, G)$ to $(X, H)$.

In the third section we consider lifting recursion properties in the special case of group actions on a covering space $\tilde{X}$ induced by the action of a group $G$ on $X$. The results tie together previous work of Markley [4] and of one of the authors [5]. The last section contains a generalization to fibre bundles with totally disconnected bundle groups.

Received by the editors February 10, 1974 and, in revised form, June 20, 1974. $54 \mathrm{C} 10$.

AMS (MOS) subject classifications (1970). Primary 54H20, 55A10; Secondary

Key words and phrases. Transformation group homomorphism, covering space, recursion, compact-covering map.

Copyright $\odot$ 1975. American Mathematical Society 
The language will be that of [3], but with the group actions written on the left rather than on the right. A topological transformation group is a continuous map $\mu: G \times X \rightarrow X$, where $X$ is a topological space and $G$ is a topological group, such that $\mu\left(g_{1}, \mu\left(g_{2}, x\right)\right)=\mu\left(g_{1} g_{2}, x\right)$ for all $x$ in $X$ and for all $g_{1}, g_{2}$ in $G$, and if $e$ is the identity element of $G$ then $\mu(e, x)=x$ for all $x$ in $X$. It may be denoted by $(X, G)$ or by $(X, G, \mu)$. Also $\mu(g, x)$ may be abbreviated to $g x$. For a fixed point $x_{0} \in X$ the map $\mu_{x_{0}}: G \rightarrow X$ is defined by $\mu_{x_{0}}(g)=\mu\left(g, x_{0}\right)$.

A transformation group homomorphism $(\phi, \psi):(Y, H, \nu) \rightarrow(X, G, \mu)$ is a continuous mapping $\phi: Y \rightarrow X$ and a homomorphism $\psi: H \rightarrow G$ such that $\phi \nu(h, y)=\mu(\psi h, \phi y)$ for all $y$ in $Y$ and for all $h$ in $H$. It is an epimorphism if both $\phi$ and $\psi$ are surjective.

\section{Lifting recursion properties through group homomorphisms.}

Definition 2.1. An intrinsic admissible property lifts through a homomorphism $\psi: H \rightarrow G$ if whenever $A$ is admissible in $G, \psi^{-1}(A \cap \psi H)$ is admissible in $H$.

Proposition 2.2. If $x$ is a recursive point of $(X, G)$ and the admissible property lifts through a homomorphism $\psi: H \rightarrow G$ then $x$ is a recursive point of $(X, H)$.

The proposition is obvious. The remaining problem is that of finding useful classes in which intrinsic admissible properties lift through homomorphisms. The concept of compact-covering maps leads to two such cases.

Definition 2.3. A continuous map $\phi: Y \rightarrow X$ is said to be compactcovering if for each compact set $K \subset X$ there exists a compact subset $K^{\prime} \subset Y$ such that $\phi K^{\prime}=K$.

The following result will be required in the next section.

Proposition 2.4. A local homeomorphism of a topological space onto a regular space is a compact-covering map.

The next result occurs in the first author's $\mathrm{Ph}$. D. thesis [1], in which the maps are called semiproper maps.

Proposition 2.5. If $\psi: H \rightarrow G$ is a compact-covering epimorphism then (right) syndetic and extensive lift through $\psi$.

Proof. Let $A$ be syndetic in $G$, and let $K$ be a compact subset of $G$ such that $K A=G$. Let $K^{\prime}$ be a compact subset of $H$ such that $\psi K^{\prime}=K$. Now given $h \in H$ there exist $a \in A, k \in K$ such that $\psi h=k a$. Choose 
$k^{\prime} \in K^{\prime} \cap \psi^{-1} k$, and set $a^{\prime}=\left(k^{\prime}\right)^{-1} h$. Then $k^{\prime} a^{\prime}=b$ and $\psi a^{\prime}=a$. Hence $\psi^{-1} A$ is syndetic in $H$.

Let $A$ be an extensive subset of $G$ and let $R$ be a replete semigroup in $H$. Then $\psi R$ is a replete semigroup in $G$ and $A \cap \psi R \neq \phi$. Consequently $\psi^{-1} A \cap R \neq \phi$, and so $\psi^{-1} A$ is extensive in $H$.

Corollary 2.6. If $\psi: H \rightarrow G$ is a compact-covering epimorphism and $x$ is \{almost periodic\} \{recurrent\} under the action of $G$ then it is $\{$ almost periodic\} \{recurrent under the action of $H$.

3. Induced actions on covering spaces. Throughout this section $X$ will be path-connected, locally path-connected and locally simply connected, and $G$ will be locally path-connected.

If in addition $G$ is path-connected and locally simply connected then the action of $G$ on $X$ induces covering actions of the covering spaces of $G$ on the covering spaces of $X$. Precisely, if $\pi^{1}$ and $\pi^{2}$ are normal subgroups of $\pi_{1}\left(X, x_{0}\right)$ and $\pi_{1}(G, e)$ such that $\mu_{x_{0} *} \pi^{2} \subset \pi^{1}$ then there is a unique action

$$
\tilde{\mu}:\left([b]_{\pi^{2}},[f]_{\pi^{1}}\right) \rightarrow\left[\mu_{x_{0}} b+b(1) f\right]_{\pi^{1}}
$$

such that the natural projections constitute an epimorphism of $\left(\tilde{X}_{\pi^{1}}, \tilde{G}_{\pi^{2}}, \tilde{\mu}\right)$ onto $(X, G, \mu)$. The deck transformations of $\tilde{X}_{\pi^{1}}$ are automorphisms of the transformation group. Thus the results of $\$ 2$ can be used to lift some recursion properties from $(X, G)$ to $\left(X, \widetilde{G}_{\pi^{2}}\right)$, while the results of Markley [4] can be used to lift them from $\left(X, \widetilde{G}_{\pi^{2}}\right)$ to $\left(\widetilde{X}_{\pi^{1}}, \tilde{G}_{\pi^{2}}\right)$. In particular, if $x$ is an almost periodic (or recurrent) point under the action of $G$ on $X$, then each point in the fibre over $x$ is almost periodic (or recurrent) under the action of $\widetilde{G}$ on $\tilde{X}$.

If $G$ is not path-connected and locally simply connected then one can turn to certain extensions of the group $G$ to obtain covering actions on covering spaces of $X$. A simpler notation than that used in [5] in which these notions were introduced will be sufficient here. For each normal subgroup $\pi^{0}$ of $\pi_{1}\left(X, x_{0}\right)$, the corresponding extension of $G$ is the group $\sigma$ whose elements are homotopy classes $[f ; g]_{\pi_{0}}$ of paths from $x_{0}$ to $g x_{0}$ in $X$. The equivalence relation is given by $(f, g) R\left(f^{\prime}, g^{\prime}\right)$ if and only if $g=g^{\prime}$ and $\left[f-f^{\prime}\right] \in \pi^{0}$, while the rule of composition is

$$
\left[f_{1} ; g_{1}\right]_{\pi^{0}} *\left[f_{2} ; g_{2}\right]_{\pi^{0}}=\left[f_{1}+g_{1} f_{2} ; g_{1} g_{2}\right]_{\pi^{0}}
$$

If $k$ is a path from $x_{0}$ to $g x_{0}$ then the map $[f] \rightarrow[k+g f-k]$ is an auto- 
morphism of $\pi_{1}\left(X, x_{0}\right)$. The image of a normal subgroup $\pi^{0}$ is a normal subgroup $g_{*} \pi^{0}$ which is independent of $k$. The normal subgroup $\pi^{0}$ is said to be invariant under $G$ if for every $g \in G, g_{*} \pi^{0}=\pi^{0}$. Note that if $G$ is pathconnected then every normal subgroup of $\pi_{1}\left(X, x_{0}\right)$ is invariant under $G$. A topology for $\sigma$ is defined in [5] in a similar way to the standard topology for $\tilde{X}$. It is shown there that if $\pi^{0} \subset \pi^{1}$ are normal subgroups of $\pi_{1}\left(X, x_{0}\right)$ which are both invariant under $G$ then the map

$$
\left([f ; g]_{\pi^{0}},\left[f^{\prime}\right]_{\pi^{1}}\right) \rightarrow\left[f+g f^{\prime}\right]_{\pi^{1}}
$$

is a transformation group action of $\sigma$ on $\tilde{X}_{\pi^{1}}$ such that the natural projections form an epimorphism onto $(X, G)$. In the remainder of this section suffixes will be omitted, but the covering space $\tilde{X}$ and the homotopy classes of loops [ $f$ ] will be relative to $\pi^{1}$ while the classes $[f ; g]$ will be relative to $\pi^{0}$.

The next proposition shows that the results of $\$ 2$ can be used to lift some recursion properties from $(X, G)$ to $(X, \sigma)$. However, Markley's methods cannot always be used in this case to lift recursion properties from $(X, \sigma)$ to $(\tilde{X}, \sigma)$ since the deck transformations of $\tilde{X}$ need not be automorphisms of the transformation group. The conditions for the deck transformations to be automorphisms, to which Markley referred in the introduction to his paper, have not yet been published and are given here.

Proposition 3.1. The projection $j: \sigma \rightarrow G, j([f ; g])=g$, is a local homeomorphism. If $G$ is locally simply connected as well as locally path-connected then $\sigma$ is a covering space of $G$.

Proof. A basis of open neighbourhoods for the topology for $\sigma$ is defined as follows. Given $[f ; g]$ and open neighbourhoods $U\left(g x_{0}\right)$ in $X$ and $V(e)$ in $G$, define $W[f ; g]$ to be the set of classes $\left[f+f^{\prime} ; g^{\prime}\right]$ where $g^{\prime} g^{-1} \in V$ and $f^{\prime}$ is a path in $U\left(g x_{0}\right)$ from $g x_{0}$ to $g^{\prime} x_{0^{*}}$. Thus the projection $j$ is continuous and open. If loops in $U\left(g x_{0}\right)$ are nullhomotopic in $X$ then the restriction of $j$ to the corresponding set $W[f ; g]$ is one-to-one. Hence $j$ is a local homeomorphism.

Now let $h$ be a path from $g$ to $g^{\prime}$ in $G$, and let $f$ be a path from $x_{0}$ to $g x_{0}$ in $X$. We define a path in $\sigma$ which is formed by stretching the path $f$ to the path $f+\mu_{x_{0}} h$. For each number $t \in I$ set

$$
\begin{array}{ll}
f_{t}: I \rightarrow X, & f_{t}: s \rightarrow \begin{cases}f(s+s t), & 0 \leq s \leq(1+t)^{-1}, \\
\mu_{x_{0}} b(s+s t-1), & (1+t)^{-1} \leq s \leq 1,\end{cases} \\
\tilde{b}: I \rightarrow \sigma, & \tilde{b}: t \rightarrow\left[f_{t}, b(t)\right] .
\end{array}
$$


Then the map $\tilde{b}$ is continuous and $\tilde{j} \tilde{h}=b$. Thus every path $h$ in $G$ can be lifted to a path in $\sigma$ whose initial point is an arbitrary point over the initial point of $h$. It follows from a theorem of Browder [2] that if $G$ is locally simply connected then $j$ is a covering map.

In order to state the conditions for deck transformations of $\tilde{X}$ to be automorphisms of $(\widetilde{X}, \sigma)$ some more notation must be introduced.

Note first that for each element $[f ; g] \in \sigma$ the map $[f ; g]_{h}:[l] \rightarrow[f+$ $g l-f]$ is an automorphism of $\pi_{1}\left(X, x_{0}\right) / \pi^{1}$, and the map $[f ; g] \rightarrow[f ; g]_{q}$ is a homomorphism of $\sigma$ to the group of automorphisms of $\pi_{1}\left(X, x_{0}\right) / \pi^{1}$. The proof is routine once it has been shown that the map $[f ; g]_{\mathrm{b}}$ is well defined. It is also easy to check that for every path $h$ from $e$ to $g$ in $G$ the map $\left[\mu_{x_{0}} h ; g\right]_{h}$ is the identity. Moreover, if $G$ is path-connected and $\pi^{1}$ contains the commutator subgroup of $\pi_{1}\left(X, x_{0}\right)$ then every automorphism $[f ; g]_{q}$ is the identity. In order to obtain a specific representation of the group of deck transformations of $\tilde{X}$ we shall assume that $\pi^{1}=p_{*} \pi_{1}\left(\tilde{X}, \tilde{x}_{0}\right)$ for some point $\tilde{x}_{0}$ in the fibre over $x_{0}$. The deck transformation corresponding to an element $[l]$ of $\pi_{1}\left(X, x_{0}\right) / \pi^{1}$ will be denoted by $[l]_{b}:[f] \rightarrow[l+f]$.

Proposition 3.2. A deck transformation $[l]_{b}$ is an automorphism of $(\tilde{X}, \sigma, \tilde{\mu})$ if and only if for every element $[f ; g] \in \sigma$ the automorphism $[f ; g]_{h}$ of $\pi_{1}\left(X, x_{0}\right) / \pi^{1}$ leaves $[l]$ invariant. Every deck transformation is an automorphism if and only if every automorphism $[f ; g]_{h}$ is the identity.

Proof. If $[f ; g] \in \sigma$ and $\left[f_{1}\right] \in \tilde{X}$ then

$$
[l]_{b} \tilde{\mu}\left([f ; g],\left[f_{1}\right]\right)=[l]_{b}\left[f+g f_{1}\right]=\left[l+f+g f_{1}\right]
$$

and

$$
\tilde{\mu}\left([f ; g],[l]_{1}\left[f_{1}\right]\right)=\left[f+g l+g f_{1}\right] .
$$

Now if every automorphism leaves $[l]$ invariant then $[f ; g]_{\natural}[l]=[f+g l-f]$ $=[l]$ and

$$
[l]_{b}\left[f+g f_{1}\right]=[f+g l-f]\left[f+g f_{1}\right]=\left[f+g l+g f_{1}\right] .
$$

Hence $[l]_{b}$ is an automorphism of the transformation group. Conversely, if $[l]_{b}$ is an automorphism, then given $[f ; g]$ we have $\left[l+f+g f_{1}\right]=[f+g l+$ $\left.g f_{1}\right]$ for all $\left[f_{1}\right]$. In particular, taking $f_{1}=-g^{-1} f$, we have $[l]=[f+g l-f]$. Hence $[l]$ is invariant under every automorphism $[f ; g]$.

Corollary 3.3. If $G$ is path-connected and $\pi_{1}\left(X, x_{0}\right)=\pi^{0} \times \mu_{x_{0 *} \pi_{1}}(G, e)$ then every deck transformation is an automorphism. If $G$ is path-connected 
and $\pi^{1}$ contains the commutator subgroup of $\pi_{1}\left(X, x_{0}\right)$ then every deck transformation is an automorphism.

Corollary 3.4. Let either of the conditions of Corollary 3.3 hold. Then if $x$ is \{almost periodic\} \{recurrent\} under the action of $G$ on $X$, each point in the fibre over $x$ is \{almost periodic\} \{recurrent $\}$ under the action of $\sigma$ on $\tilde{X}$.

4. Induced actions on fibre bundles. An action of a group $G$ on the base space $X$ of a fibre bundle with totally disconnected bundle group induces covering actions of covering groups of $G$ on the bundle space $B$. In this case also the results of $\$ 2$ can be used to lift some recursion properties from $(X, G)$ to $(X, \tilde{G})$. While Markley's results cannot be used, his arguments admit a generalization which can in some cases be used to lift recursion properties from $(X, \widetilde{G})$ to $(B, \widetilde{G})$.

Let $p: B \rightarrow X$ be a bundle map for which the bundle group is totally disconnected. Let $f$ be a path from $x$ to $x^{\prime}$ in $X$. Then there is a unique translation of the fibre over $x$ along the path $f$. Each point in the fibre over $x$ is translated along a path $\tilde{f}$ in $B$ which lies over the path $f$. If $\pi^{1}$ is the kernel of a characteristic homomorphism $\chi: \pi_{1}\left(X, x_{0}\right) \rightarrow G$, then the terminal point $b^{\prime}$ of the path $\tilde{f}$ depends only on the homotopy class $[f]_{\pi^{1}}$.

Now let $(X, G, \mu)$ be a transformation group for which $G$ is path-connected, locally path-connected and locally simply connected. Suppose that $\pi^{2}$ is a subgroup of $\pi_{1}(G, e)$ such that $\mu_{x_{0 *}} \pi^{2} \subset \pi^{1}$. Then if $h$ is a path from $e$ to $g$ in $G, b \in B$, and $\mu_{p_{b}} b=f$, the endpoint $b^{\prime}$ of the path $\tilde{f}_{b}$ depends only on the homotopy class $[b]_{\pi}$. Thus the map

$$
\nu: \widetilde{G}_{\pi^{2}} \times B \rightarrow B, \quad \nu\left([b]_{\pi^{2}}, b\right)=b^{\prime}
$$

is well defined.

Proposition 4.1. If the base space $X$ of the bundle is paracompact and Hausdorff then with the map $\nu$ just defined $\left(B, \widetilde{G}_{\pi^{2}}, \nu\right)$ is a transformation group. The natural projections constitute an epimorphism of this transformation group to the transformation group $(X, G, \mu)$.

The covering homotopy property ensures that $\nu$ is continuous. The other conditions are clearly fulfilled.

Markley's results on lifting recursion properties refer to recursion with respect to classes of admissible subsets which satisfy the following hypothesis. He proves that the class of syndetic sets and the class of extensive sets of a group satisfy the hypothesis. While his results refer explicitly to 
group actions on regular covering spaces, the basic property which he uses in the second section of [4] is that if two points lie in the same fibre then their orbit closures are either equal or disjoint. This condition and the other conditions of the next proposition are satisfied for induced covering group actions on a bundle space in which the base space is locally compact, the fibre is compact and the bundle group is finite.

Definition 4.2. A class $\mathcal{Q}$ of admissible subsets of a group $G$ satisfies the decomposition hypothesis if given any decomposition $A_{1}, \ldots, A_{n}$ of a set $A$ in $\mathbb{Q}$ and $g_{1}, \ldots, g_{n}$ in $G$, then $\bigcup_{i=1}^{n} g_{i} A_{i}$ is in $\mathbb{A}$.

Proposition 4.3. Let $(X, G)$ and $(Y, G)$ be transformation groups, and let $\phi: Y \rightarrow X$ be an equivariant open map such that if $\phi y_{1}=\phi y_{2}$ then either $\overline{G y_{1}}=\overline{G y_{2}}$ or $\overline{G y_{1}} \cap \overline{G y_{2}}=\phi$. Suppose that there exists a point $x$ of $X$ which has a neighbourhood $W(x)$ such that $\phi^{-1} \bar{W}$ is compact, and a point $y_{1}$ of $\phi^{-1} x$ such that $\overline{G y_{1}} \cap \phi^{-1} x$ is finite. Let $\mathbb{Q}$ be a class of admissible subsets satisfying the decomposition hypothesis. Then $x$ is a recursive point of $(X, G)$ if and only if $y_{1}$ is a recursive point of $(Y, G)$.

Proof. The sufficiency of the condition is immediate since the map is open. Now let $x$ be recursive, let $\overline{G y_{1}} \cap \phi^{-1} x=\left\{y_{1}, y_{2}, \ldots, y_{n}\right\}$, and let $U_{1}\left(y_{1}\right)$ be a neighbourhood of $y_{1}$. Since $\overline{G y_{i}}=\overline{G y_{1}}$ whenever $1 \leq i \leq n$ there exist elements $g_{i}$ of $G$ and neighbourhoods $U_{i}\left(y_{i}\right)$ such that $g_{i} U_{i} \subset U_{1}$. Because of the compactness of $\phi^{-1} \bar{W}$ there exists a neighbourhood $V \subset$ $\bigcap_{i=1}^{n} \phi U_{i} \cap W$ such that $\left(\phi^{-1} V-\bigcup_{i=1}^{n} U_{i}\right) \cap \overline{G y_{1}}=\phi$. There exists a subset $A \in \mathbb{Q}$ such that $A x \subset V$. Given $x \in A$ we have $g y_{1} \in U_{i}$ for some $i \leq n$. Then $g_{i} g y_{1} \in U_{1}$. Let $A_{i}=\left\{g \mid g y_{1} \notin U_{1}, \ldots, U_{i-1} ; g y_{1} \in U_{i}\right\}$ and $A^{\prime}=$ $\bigcup_{i=1}^{n} g_{i} A_{i}$. Then $A^{\prime} y_{1} \in U_{1}$ and $A^{\prime} \in \mathbb{Q}$. Hence $y_{1}$ is recursive.

Unfortunately, the corresponding result for local recursion requires the additional conditions that $Y$ is locally connected and $\phi^{-1} x$ is finite, and so is essentially the same as the result proved by Markley.

Corollary 4.4. Let $B$ be a fibre bundle whose base space $X$ is locally compact and paracompact, whose fibre is compact and whose bundle group is finite. Let $(X, G)$ be a transformation group and $(B, \widetilde{G})$ be an induced covering transformation group. If $x \in X$ is $\{$ almost periodic\} \{recurrent $\}$ under the action of $G$ then each point of the fibre over $x$ is \{almost periodic\} \{recurrent\} under the action of $\widetilde{G}$.

\section{REFERENCES}

1. S. H. Al-Kutaibi, Transferent and referent properties, Ph.D. Thesis, University of Southampton, 1971. 
2. F. E. Browder, Covering spaces, fibre spaces and local homeomorphis ms, Duke Math. J. 21 (1954), 329-336. MR 15, 978.

3. W. H. Gottschalk and G. A. Hedlund, Topological dynamics, Amer. Math. Soc. Colloq. Publ., vol. 36, Amer. Math. Soc., Providence, R.I., 1955. MR 17, 650.

4. N. G. Markley, Lifting dynamical properties, Math. Systems Theory 5 (1971), 299-305. MR $46 \# 4514$.

5. F. Rhodes, On lifting transformation groups, Proc. Amer. Math. Soc. 19 (1968), 905-908. MR 38 \#5196.

DEPARTMENT OF MATHEMATICS, UNIVERSITY OF BASRAH, BASRAH, IRAQ

DEPARTMENT OF MATHEMATICS, UNIVERSITY OF SOUTHAMPTON, HIGHFIELD, SOUTHAMPTON, GREAT BRITAIN 